\title{
Multi-State Density Functional Theory for Ground and Excited States
}

\author{
Yangyi $\mathrm{Lu}^{*}$ \\ Institute of Systems and Physical Biology, Shenzhen Bay Laboratory, Shenzhen 518055, China \\ Jiali $\mathrm{Gao}^{\dagger}$ \\ Institute of Systems and Physical Biology, Shenzhen Bay Laboratory, \\ Shenzhen 518055, China; Department of Chemistry and Supercomputing Institute, \\ University of Minnesota, Minneapolis, Minnesota 55455, United States
}

(Dated: October 12, 2021)

\begin{abstract}
We report a rigorous formulation of multi-state density functional theory (MSDFT) that extends the Kohn-Sham (KS) energy functional for the ground state to a Hamiltonian matrix functional $\mathcal{H}[\mathcal{D}]$ of the density matrix $\mathcal{D}$ in the space spanned by the lowest $N$ adiabatic states. We establish a variational principle of MSDFT, which guarantees that the variational optimization results in a Hamiltonian matrix, whose eigenvalues are the lowest $N$ eigen-energies of the system. We present an explicit expression of $\mathcal{H}[\mathcal{D}]$ and introduce the correlation matrix functional, $\mathcal{E}_{c}[\mathcal{D}]$. Akin to KS-DFT for the ground state, a universal multi-state correlation potential $v_{\mathrm{MS}}^{c}(r)$ is derived for a two-state system as an illustrative example. This work shows that MSDFT is an exact density functional theory that treats the ground and excited states on an equal footing and provides a framework for practical applications and future developments of approximate functionals for excited states.
\end{abstract}

Introduction The Hohenberg-Kohn theorems establish the existence of the ground-state energy as a functional of the density[1], whereas the Kohn-Sham density functional theory (KS-DFT) provides a practical approach for computing the energy and properties by introducing one-body orbitals[2-4]. For electronically excited states, linear-response time-dependent density functional theory (TDDFT) is typically used[5-7]. These methods have now become indispensable tools for electronic structure calculations of molecules and materials[8-11]. However, there are also well-known difficulties in the current density functional approximations, including the treatment of systems with degeneracy or near degeneracy and excitations containing charge-transfer character[12] Consequently, the quest for a time-independent approach in DFT for excited states has long been a goal of theorists. To this end, the ensemble density functional theory (EDFT) of Theophilou[13] and the state-weighted extension by Gross, Oliveira and Kohn (GOK) established a rigorous foundation[14-17], but these theorems only provided the average energy of an ensemble of $\mathrm{N}$-states (ensemble energy) without the specific energies and vectors of individual states. As a result, excitation energies must be extracted indirectly with additional calculations and different ensembles. Critically, both Theophilou's theorem and the GOK alternative do not formulate a procedure for constructing the energy functionals of the individual states, although significant progress has been made recently in the understanding of the properties of ensemble density functionals[18-20] in the framework of Kohn-Sham theory[12, 21, 22].

Building up on the work of Theophilou[13], we introduce two theorems that establish the Hamiltonian

\footnotetext{
*luyy@szbl.ac.cn

$\dagger$ gao@jialigao.org
}

as a matrix functional $\mathcal{H}[\mathcal{D}]$ of the density matrix $\mathcal{D}$ and a variational theory for determining the state energies and vectors in the subspace of the lowest $\mathrm{N}$ eigenstates. Furthermore, we introduce a multistate interacting system to determine the exact state densities and ensemble density, akin to, but fundamentally different from, the Kohn-Sham non-interacting reference for the single, ground-state density. Importantly, the tremendous success of the approximate density functionals developed for KS-DFT can be directly used[23]. In fact, such a multistate density functional theory (MSDFT) has been used in the form of nonorthogonal state interaction (NOSI) in a variety of applications[24, 25], including valence and charge-transfer excited states[26-28], corelevel excitation of closed and open-shell molecules[29], singlet-fission[30] and proton-coupled electron transfer reactions[31] in systems of chemical, biological and materials interest. Here, we distinguish the term state interaction from configuration interaction because the former describes interacting states that include dynamic correlation, whereas the latter represents determinant configuration interaction $[32,33]$. In this Letter, we also derive an exact expression of the Hamiltonian matrix functional and illustrate the results for a two-state system in terms of local density-matrix approximation.

We consider a system of $n_{e}$ electrons under the local multiplicative external potential $\hat{V}_{\mathrm{ext}}=\sum_{j=1}^{n_{e}} v\left(r_{j}\right)$, where the Hamiltonian is given by $\hat{H}=\hat{H}_{0}+\hat{V}_{\text {ext }}$ and $\hat{H}_{0}$ consists of the kinetic and electronic Coulomb terms,

$$
\hat{H}_{0}=\sum_{j=1}^{n_{e}} \frac{p_{j}^{2}}{2 m}+\frac{e^{2}}{4 \pi \epsilon_{0}} \sum_{i>j}^{n_{e}} \frac{1}{\left|r_{i}-r_{j}\right|}
$$

In Eq. (1), $r_{j}$ and $p_{j}$ are, respectively, the coordinate and momentum of electron $j, e$ and $m$ are the electronic charge and mass, and $\epsilon_{0}$ is the vacuum permittivity. 
Ensemble density functional theory In order to introduce the matrix functional for the Hamiltonian $\hat{H}$, we first review the ensemble DFT of Theophilou. We denote the ensemble expectation of an operator $\hat{O}$ as $\operatorname{tr}_{S}(\hat{O})=$ $N^{-1} \sum_{A=1}^{N}\left\langle\Phi_{A}|\hat{O}| \Phi_{A}\right\rangle$, where $\left\{\Phi_{A}, A=1, \cdots, N\right\}$ is an arbitrary orthonormal basis in an $N$-dimensional subspace $\mathbb{S}^{N}$ of the complete Hilbert space of the system $\mathbb{H}, \mathbb{S}^{N} \subset \mathbb{H}$. Throughout this Letter, we use $I, J, \cdots$ to denote eigenstates and $A, B, \cdots$ for general basis states. We assume that all vectors in $\mathbb{H}$ are normalized.

Extending the Hohenberg-Kohn theorem for the ground state[1], Theophilou proved that given the number of states $N_{s} \geq 1$ and an external potential $\hat{V}_{\text {ext }}$, there exists a unique $N_{s}$-dimensional subspace $\mathbb{V}_{\text {min }}^{N_{s}} \subset \mathbb{H}$ that minimizes the ensemble energy of the system[13],

$$
\mathbb{E}[v]=\operatorname{tr}_{V}(\hat{H})=\min \left\{\operatorname{tr}_{S}(\hat{H}) \mid \mathbb{S}^{N_{s}} \subset \mathbb{H}\right\}
$$

The unique subspace $\mathbb{V}_{\min }^{N_{s}}$, spanned by the lowest $N_{s}$ eigenstates of $\hat{H}, \hat{H} \Psi_{I}=E_{I} \Psi_{I}$ with $E_{1} \leq \cdots \leq E_{N_{s}}$, including all degenerate eigenstates of the highest eigenvalue $E_{N_{s}}$, corresponds to a unique ensemble density $\rho_{V}^{0}=\operatorname{tr}_{V}(\hat{\rho})$ with $\hat{\rho}=\sum_{j}^{n_{e}} \delta\left(r-r_{j}\right)$ being the density operator. $\mathbb{E}[v]=N_{s}^{-1} \sum_{I=1}^{N_{s}} E_{I}$ is the minimal ensemble energy.

Ensemble Levy-Lieb functional Further insights are provided by generalizing the Levy-Lieb functional to the ensemble energy. We define the Levy-Lieb functional for an arbitrary ensemble density $\rho_{V}$ as [34-36],

$$
\mathfrak{F}_{L}\left[\rho_{V}\right]=\inf \left\{\operatorname{tr}_{S}\left(\hat{H}_{0}\right) \mid \operatorname{tr}_{S}(\hat{\rho})=\rho_{V}, \forall \mathbb{S}^{N_{s}} \subset \mathbb{H}\right\}
$$

Analogous to the ground-state functional, the infimum of the ensemble energy functional $\mathfrak{F}_{L}\left[\rho_{V}\right]$ is also a minimum (see Supplementary Note 1). Therefore, provided $\rho_{V}$, one can always find a subspace that gives $\mathfrak{F}_{L}\left[\rho_{V}\right]$, and then all the theorems in the original work of Lieb for DFT can be applied to the generalized ensemble functional $\mathfrak{F}_{L}\left[\rho_{V}\right]$. A direct result of Theorem $3.10 \mathrm{in} \mathrm{Lieb[35]} \mathrm{is} \mathrm{the} \mathrm{variational}$ principle in terms of the functional $\mathfrak{F}_{L}\left[\rho_{V}\right]$,

$$
\mathbb{E}[v]=\inf \left\{\mathfrak{E}\left[\rho_{V}\right]\right\}
$$

where $\mathfrak{E}\left[\rho_{V}\right]=\mathfrak{F}_{L}\left[\rho_{V}\right]+\int \mathrm{d} r v(r) \rho_{V}(r)$ is the ensemble energy functional of $\rho_{V}$, and $\mathfrak{E}\left[\rho_{V}\right]=\mathbb{E}[v]$ when $\rho_{V}=\rho_{V}^{0}$.

Multi-state density functional theory With the above preliminaries, we are ready to establish the Hamiltonian matrix functional and the variational principle of MSDFT.

Given $N_{s}$ and $\rho_{V}$, the functional $\mathfrak{F}_{L}\left[\rho_{V}\right]$ (Eq.3) defines an $N_{s}$-dimensional subspace, denoted as $\mathbb{V}\left[\rho_{V}\right] \subset \mathbb{H}$. For an arbitrary basis, i.e., a linearly independent set of states, $\left\{\Phi_{A}\right\} \in \mathbb{V}\left[\rho_{V}\right]$, we define the multi-state density matrix $\mathcal{D}$ with the $(A, B)$-th element, $\mathcal{D}_{A B}=\left\langle\Phi_{A}|\hat{\rho}| \Phi_{B}\right\rangle$.

Theorem 1 : Given the number of states $N_{s}$, there exists a density matrix of degree $N_{s}, \mathcal{D}$, such that a unique ensemble density $\rho_{V}$ is determined. Then, the Hamiltonian projected in the subspace $\mathbb{V}\left[\rho_{V}\right]$ is a matrix functional of $\mathcal{D}$, denoted as $\mathcal{H}[\mathcal{D}]$.

Proof: To prove this theorem, we first establish that given the number of states $N_{s}$, there is a one-to-one correspondence between a density matrix $\mathcal{D}$ and a set of $N_{s}$ linearly independent states, $\left\{\Phi_{A}\right\} \in \mathbb{H}$, which is a basis of $\mathbb{V}\left[\rho_{V}\right]$. The details of the proof are given in Supplementary Note 2. Then, the ensemble density is given by $\rho_{V}=\operatorname{tr}\left(\mathcal{S}^{-1} \mathcal{D}\right)$, where $\mathcal{S}$ is the overlap matrix uniquely determined by $\mathcal{D}$. We emphasize that, in general, the states in $\left\{\Phi_{A}\right\}$ are non-orthogonal.

Once this one-to-one correspondence is established. Then, given $\mathcal{D}$, whose ensemble density is $\rho_{V}$, there is a basis $\left\{\Phi_{A}\right\} \in \mathbb{V}\left[\rho_{V}\right]$. The Hamiltonian projected in $\mathbb{V}\left[\rho_{V}\right]$ is uniquely determined by $\left\{\Phi_{A}\right\}$ with its matrix elements, $H_{A B}=\left\langle\Phi_{A}|\hat{H}| \Phi_{B}\right\rangle$. Therefore, the Hamiltonian is a matrix functional of $\mathcal{D}$. The proof is complete.

We can see from the proof that there are infinite density matrices that satisfy Theorem 1 . Given $\mathcal{D}$, we compute $\mathcal{H}[\mathcal{D}]$, which can be diagonalized by a matrix $\mathcal{C}$ belonging to the Special Linear group of degree $N_{s}$, $\mathrm{SL}\left(N_{s}, \mathbb{C}\right)[37]$. We define the multi-state energy functional as the ensemble energy of the space $\mathbb{V}\left[\rho_{V}\right]$, which is the average of the eigenvalues of $\mathcal{H}[\mathcal{D}]$,

$$
E_{\mathrm{MS}}[\mathcal{D}]=N_{s}^{-1} \sum_{I}^{N_{s}} \sum_{A, B}^{N_{s}} \mathcal{C}_{I A} \mathcal{C}_{I B}^{*} \mathcal{H}_{A B}=\operatorname{tr}\left(\mathcal{C H}[\mathcal{D}] \mathcal{C}^{\dagger}\right)
$$

with $\mathcal{H}_{A B}$ being the $(A, B)$-th element of $\mathcal{H}[\mathcal{D}]$.

Given Eq. 5, we arrive at the following theorem.

\section{Theorem 2 (Variational principle of MSDFT) :}

$$
\mathbb{E}[v]=\inf \left\{E_{\mathrm{MS}}[\mathcal{D}]\right\}
$$

which is equivalent to the variational principle of Theophilou (Eq.4) with $\mathbb{E}[v]$ given in terms of the Hamiltonian matrix functional (Eq.5). Moreover, the eigenvalues of the Hamiltonian matrix functional are exactly the $N_{s}$ lowest adiabatic energies of the system, $\left\{E_{1}, \cdots, E_{N_{s}}\right\}$. 
Proof : Since $E_{\mathrm{MS}}[\mathcal{D}]$ (Eq.5) is defined as the ensemble energy over the subspace $\mathbb{V}\left[\rho_{V}\right]$ with $\rho_{V}$ determined by $\mathcal{D}$, it is equal to the ensemble energy functional $\mathfrak{E}\left[\rho_{V}\right]$ defined on the same space $\mathbb{V}\left[\rho_{V}\right]$,

$$
E_{\mathrm{MS}}[\mathcal{D}]=\mathfrak{E}\left[\rho_{V}\right]
$$

The variational principle of MSDFT (Eq.6) then follows directly from that of Theophilou (Eq.4),

$$
\mathbb{E}[v]=\inf \left\{\mathfrak{E}\left[\rho_{V}\right]\right\}=\inf \left\{E_{\mathrm{MS}}[\mathcal{D}]\right\}
$$

We minimize $E_{\mathrm{MS}}[\mathcal{D}]$ by varying the elements of $\mathcal{D}$ until we reach $\mathbb{E}[v]$ with an optimal density matrix $\mathcal{D}^{o}$ that gives the ensemble density $\rho_{V}^{0}$. According to Theorem $1, \mathcal{D}^{o}$ corresponds to a unique basis $\left\{\Phi_{A}^{o}\right\} \in \mathbb{V}_{\text {min }}^{N_{s}}$, where $\mathbb{V}_{\min }^{N_{s}}=\operatorname{span}\left\{\Psi_{1}, \cdots, \Psi_{N_{s}}\right\}$, with $\Psi_{I}$ being the $I$ th eigenstate of $\hat{H}$ with energy $E_{I}$. Since the Hamiltonian matrix in $\mathbb{V}_{\min }^{N_{s}}$ is constructed by $\left\{\Phi_{A}^{o}\right\}$, which are linear combinations of $\left\{\Psi_{I}\right\}$, the eigenvalues of the Hamiltonian matrix functional are necessarily the lowest $N_{s}$ eigenenergies of the system and $\mathbb{E}[v]=N_{s}^{-1} \sum_{I}^{N_{s}} E_{I}$. The proof is then complete.

Transformation property of the Hamiltonian matrix functional According to Theorem 1, given a density matrix, $\mathcal{D}$, there is a unique basis $\left\{\Phi_{A}\right\} \in \mathbb{V}\left[\rho_{V}\right]$ with $\rho_{V}$ determined by $\mathcal{D}$. Any linear transformation of the basis that belongs to $\mathrm{SL}\left(N_{s}, \mathbb{C}\right)$ gives another basis of $\mathbb{V}\left[\rho_{V}\right][37],\left\{\Phi_{A}^{(2)}\right\}$,

$$
\Phi_{A}^{(2), *}=\sum_{B=1}^{N_{s}} \mathcal{L}_{A B} \Phi_{B}^{*} \Rightarrow \mathcal{D}^{(2)}=\mathcal{L} \mathcal{D} \mathcal{L}^{\dagger}
$$

which induces a transformation between the corresponding density matrices, $\mathcal{D}^{(2)}$ and $\mathcal{D}$. The matrix functional $\mathcal{H}\left[\mathcal{D}^{(2)}\right]$ must also be related to that of $\mathcal{H}[\mathcal{D}]$ through $[38]$,

$$
\mathcal{H}\left[\mathcal{D}^{(2)}\right]=\mathcal{L} \mathcal{H}[\mathcal{D}] \mathcal{L}^{\dagger}
$$

Multi-state auxiliary system The Hamiltonian matrix functional can be divided into three parts,

$$
\mathcal{H}[\mathcal{D}]=\mathcal{T}[\mathcal{D}]+\mathcal{E}_{\text {Coul }}[\mathcal{D}]+\int \mathrm{d} r v(r) \mathcal{D}(r)
$$

where the three terms on the right-hand-side are, respectively, the matrix functional of kinetic energy, the electronic Coulomb energy, and the energy from external potential.

In analogy to the Kohn-Sham auxiliary system for the ground state, we introduce a set of $N_{s}$ Slater determinants $\left\{\Phi_{A}^{\mathrm{MS}}\right\}$ that form a minimal active space to yield the exact multistate (MS) density matrix $\mathcal{D}$ of the subspace $\mathbb{V}_{\min }^{N_{s}}$,

$$
\Phi_{A}^{\mathrm{MS}}\left(r_{1}, \cdots, r_{n_{e}}\right)=\left(n_{e} !\right)^{-\frac{1}{2}} \mathcal{A}\left\{\phi_{1}^{A}\left(r_{1}\right) \cdots \phi_{n_{e}}^{A}\left(r_{n_{e}}\right)\right\}
$$

where $\mathcal{A}$ is the anti-symmetrizer. The orbitals in each MS configuration are assumed to be orthonormal, $\left\langle\phi_{i}^{A} \mid \phi_{j}^{A}\right\rangle=$ $\delta_{i j}$, but no restriction is imposed between orbitals in different MS determinants. Therefore, the basis determinants in the active space are generally nonorthogonal. We then construct the density matrix element by

$$
\mathcal{D}_{A B}=\left\langle\Phi_{A}^{\mathrm{MS}}|\hat{\rho}| \Phi_{B}^{\mathrm{MS}}\right\rangle=\sum_{j, k}^{n_{e}} f_{j k}^{A B} \phi_{j}^{A, *} \phi_{k}^{B}
$$

where the coefficient $f_{j k}^{A B}$ is the overlap between two Slater determinants of $\left(n_{e}-1\right)$ orbitals (eq (13) of Supplementary Note 3 ). When $A=B$, the basis-state density is $\mathcal{D}_{A A}=\sum_{j}\left|\phi_{j}^{A}\right|^{2}$. Then the ensemble density is given by $\rho_{V}=\operatorname{tr}\left(\mathcal{S}^{-1} \mathcal{D}\right)$ with $\mathcal{S}$ being the overlap matrix, $\mathcal{S}_{A B}=\left\langle\Phi_{A}^{\mathrm{MS}} \mid \Phi_{B}^{\mathrm{MS}}\right\rangle=n_{e}^{-1} \int \mathrm{d} r \mathcal{D}_{A B}(r)[39]$.

Within the active space, $\operatorname{span}\left\{\Phi_{A}^{\mathrm{MS}}\right\}$, we define the kinetic energy matrix functional $\mathcal{T}_{s}$ with

$$
\mathcal{T}_{s}^{A B}=\left\langle\Phi_{A}^{\mathrm{MS}}\left|-\frac{\hbar^{2}}{2 m} \sum_{j=1}^{n_{e}} \nabla_{j}^{2}\right| \Phi_{B}^{\mathrm{MS}}\right\rangle=\sum_{j, k}^{n_{e}} f_{j k}^{A B} t_{j k}^{A B}
$$

where $t_{j k}^{A B}=-\frac{\hbar^{2}}{2 m}\left\langle\phi_{j}^{A}\left|\nabla^{2}\right| \phi_{k}^{B}\right\rangle$. In particular, for each diagonal element, we have a similar expression as that of KS-DFT for the ground state, $\mathcal{T}_{s}^{A A}=\sum_{j} t_{j j}^{A A}$ with $t_{j j}^{A A}=-\frac{\hbar^{2}}{2 m}\left\langle\phi_{j}^{A}\left|\nabla^{2}\right| \phi_{j}^{A}\right\rangle$. However, $\mathcal{T}_{s}^{A B}(A \neq B)$ is absent in KS-DFT.

Similarly, within $\operatorname{span}\left\{\Phi_{A}^{\mathrm{MS}}\right\}$, we define the Coulomb interaction matrix $\mathcal{E}_{\mathrm{Hx}}$ with

$$
\begin{aligned}
\mathcal{E}_{\mathrm{Hx}}^{A B} & =\left\langle\Phi_{A}^{\mathrm{MS}}\left|\frac{e^{2}}{8 \pi \epsilon_{0}} \sum_{i \neq j} \frac{1}{\left|r_{i}-r_{j}\right|}\right| \Phi_{B}^{\mathrm{MS}}\right\rangle \\
& =\sum_{i<j} \sum_{k<l} f_{i j, k l}^{A B}\langle i j|| k l\rangle_{A B}
\end{aligned}
$$

where $\langle i j|| k l\rangle_{A B}$ is the two-particle integral with $f_{i j, k l}^{A B}$ being the coefficient (eqs (15-16) of Supplementary Note 3). The diagonal element of $\mathcal{E}_{\mathrm{Hx}}$ is $\mathcal{E}_{\mathrm{Hx}}^{A A}=\sum_{i<j}\langle i j \| i j\rangle_{A A}$. From the definitions of $\mathcal{T}_{s}$ and $\mathcal{E}_{\mathrm{Hx}}$, it follows that they both satisfy the transformation property (Eq.10).

Finally, the multistate correlation matrix functional $\mathcal{E}_{c}[\mathcal{D}]$ can be defined as follows

$$
\mathcal{E}_{c}[\mathcal{D}]=\left(\mathcal{T}[\mathcal{D}]-\mathcal{T}_{s}\right)+\left(\mathcal{E}_{\text {Coul }}[\mathcal{D}]-\mathcal{E}_{\mathrm{Hx}}\right)
$$

where the energies in parentheses represent, respectively, the difference in kinetic energy and in Coulomb energy between the real (fully interacting) system and the auxiliary active space (partially interacting). Therefore, the correlation matrix functional represents the part of correlation energy not included in the active space. Then, the Hamiltonian matrix functional of MSDFT has the following generic form (eq 11),

$$
\mathcal{H}[\mathcal{D}]=\mathcal{T}_{s}+\mathcal{E}_{\mathrm{Hx}}+\int \mathrm{d} r v(r) \mathcal{D}(r)+\mathcal{E}_{c}[\mathcal{D}]
$$

The correlation matrix functional $\mathcal{E}_{c}[\mathcal{D}]$ must possess the following properties. 
1. The linear transformation property of Eq.10 is satisfied.

2. When $N_{s}=1$, the density matrix $\mathcal{D}$ becomes the single-state density $\rho_{0}$. The Hamiltonian matrix functional $\mathcal{H}[\mathcal{D}]$ is reduced to the KohnSham energy functional $E_{\mathrm{KS}}\left[\rho_{0}\right]$. If we denote the single-state electrostatic energy as $E_{H}\left[\rho_{0}\right]=$ $\frac{e^{2}}{8 \pi \epsilon_{0}} \int r_{12}^{-1} \rho_{0}\left(r_{1}\right) \rho_{0}\left(r_{2}\right), \quad \mathcal{E}_{c}[\mathcal{D}]$ becomes the $\mathrm{KS}$ exchange-correlation functional $E_{\mathrm{xc}}\left[\rho_{0}\right]$ :

$$
\mathcal{E}_{\mathrm{Hx}}+\mathcal{E}_{c}[\mathcal{D}] \rightarrow E_{H}\left[\rho_{0}\right]+E_{\mathrm{xc}}\left[\rho_{0}\right]
$$

when $\mathcal{D} \rightarrow \rho_{0}$.

3. In the full CI limit $N_{s}=N_{\mathrm{FCI}}$, where the configurations $\left\{\Phi_{A}^{\mathrm{MS}}\right\}$ form a complete basis of the full Hilbert space, the exact kinetic energy functional equals $\mathcal{T}_{s}, \mathcal{T}[\mathcal{D}]=\mathcal{T}_{s}$, and $\mathcal{E}_{\text {Coul }}[\mathcal{D}]=\mathcal{E}_{\mathrm{Hx}}$. The correlation energy functional vanishes,

$$
\mathcal{E}_{c}[\mathcal{D}]=0 \text {, when } N_{s}=N_{\mathrm{FCI}}
$$

With an explicit expression of the Hamiltonian matrix functional (Eq.17), the multi-state energy functional $E_{\mathrm{MS}}$ (Eq.5) reads

$$
E_{\mathrm{MS}}[\mathcal{D}]=\operatorname{tr}\left[\mathcal{S}^{-1} \mathcal{H}[\mathcal{D}]\right]
$$

Significantly, when $E_{\mathrm{MS}}[\mathcal{D}]=\mathbb{E}[v]$ (Theorem 2), solution of the generalized secular equation of the Hamiltonian matrix functional[40],

$$
\mathcal{H}[\mathcal{D}] \mathcal{C}=\mathcal{S C E}_{0}
$$

gives the $N_{s}$-lowest adiabatic energies $\mathcal{E}_{0}=$ $\operatorname{diag}\left(E_{1}, \cdots, E_{N_{s}}\right)$ and state vectors that give the exact densities $\left\{\rho_{I}\right\}$ of the individual eigenstates.

Local density-matrix approximation Here we derive an analytical expression for $\mathcal{E}_{c}[\mathcal{D}]$ under the local densitymatrix approximation (LDMA),

$$
\mathcal{E}_{c}^{\mathrm{LDMA}}[\mathcal{D}]=E_{0} \int \mathrm{d} r \mathcal{G}(\mathcal{D}(r))
$$

where $E_{0}$ is a real constant. In general, the correlation functional has a non-linear dependence with $\mathcal{D}$. A matrix integral transformation is required. The matrix function $\mathcal{G}(\mathcal{D}(r))$ is the correlation energy density defined by

$$
\mathcal{G}(\mathcal{D}(r))=\mathcal{S}^{\frac{1}{2}} g\left(\mathcal{D}^{\perp}(r)\right) \mathcal{S}^{\frac{1}{2}}
$$

where $\mathcal{S}$ is the overlap matrix determined by $\mathcal{D}(r)$, and $g\left(\mathcal{D}^{\perp}(r)\right)$ is a generalized matrix function of the standard Kohn-Sham correlation energy (below). $\mathcal{D}^{\perp}(r)$ is defined as $\mathcal{D}^{\perp}(r)=\mathcal{S}^{-\frac{1}{2}} \mathcal{D}(r) \mathcal{S}^{-\frac{1}{2}}$. Since for all $r \in \mathbb{R}^{3}$, the matrix $\mathcal{D}^{\perp}(r)$ is Hermitian and positive definite, we can always find a local unitary transformation with the unitary matrix $\mathcal{U}(r)$ that diagonalizes $\mathcal{D}^{\perp}(r)$,

$$
\mathcal{D}^{\perp}(r)=\mathcal{U}(r) \Lambda(r) \mathcal{U}^{\dagger}(r)
$$

with $\Lambda(r)=\operatorname{diag}\left(\lambda_{1}(r), \cdots, \lambda_{N_{s}}(r)\right)$ in which all $\lambda_{j}(r)>$ 0 . The diagonal matrix $\Lambda(r)$ is invariant under the linear transformation of $\mathcal{D}(r)$ (For proof, see Supplementary Note 4$)$. The function $g(z)$ with $z \in \mathbb{C}$ in Eq.23 can be an arbitrary analytic function within a domain $D(g) \subseteq \mathbb{C}$. The matrix function $g\left(\mathcal{D}^{\perp}(r)\right)$ is given by (For derivation, see Supplementary Note 5[41])

$g\left(\mathcal{D}^{\perp}(r)\right)=\mathcal{U}(r)\left(\begin{array}{ccc}g\left(\lambda_{1}(r)\right) & \ldots & 0 \\ \vdots & g\left(\lambda_{j}(r)\right) & \vdots \\ 0 & \cdots & g\left(\lambda_{N s}(r)\right)\end{array}\right) \mathcal{U}^{\dagger}(r)$

Eq.25 shows that both diagonal and off-diagonal elements of the correlation matrix functional can be determined using a standard correlation functional developed for KSDFT (as $g(z))$ followed by a local unitary transformation (Supplementary Note 5). By following a similar procedure, it is straightforward to include the dependence of the gradient of density matrix and kinetic-energy density matrix into $\mathcal{E}_{c}[\mathcal{D}][42,43]$.

Example Finally, we consider a special case consisting of two states $\left(N_{s}=2\right)$, where analytical expressions both for $\mathcal{U}(r)$ and $\Lambda(r)$ can be derived. For simplicity, we assume that all functions are real and the constant $E_{0}=$ 1 in Eq.22. The density matrix $\mathcal{D}$ reads

$$
\mathcal{D}(r)=\left(\begin{array}{cc}
\rho_{1}(r) & \rho_{12}(r) \\
\rho_{12}(r) & \rho_{2}(r)
\end{array}\right) \Rightarrow \mathcal{S}=\left(\begin{array}{cc}
1 & \Delta \\
\Delta & 1
\end{array}\right)
$$

which gives the overlap matrix $\mathcal{S}$ with $\Delta=$ $n_{e}^{-1} \int \mathrm{d} r \rho_{12}(r) . \mathcal{D}^{\perp}(r)$ is then given by

$$
\left(\begin{array}{cc}
\rho_{V}+\frac{\rho_{1}-\rho_{2}}{2 \sqrt{1-\Delta^{2}}} & \rho_{12}-\Delta \rho_{V} \\
\rho_{12}-\Delta \rho_{V} & \rho_{V}-\frac{\rho_{1}-\rho_{2}}{2 \sqrt{1-\Delta^{2}}}
\end{array}\right)
$$

Diagonalization of $\mathcal{D}^{\perp}(r)$ leads to $\Lambda(r)=$ $\operatorname{diag}\left(\lambda_{1}(r), \lambda_{2}(r)\right)$, where $\lambda_{1,2}(r)=\rho_{V} \pm \sqrt{\rho_{V}^{2}-\left\|\mathcal{D}^{\perp}\right\|}>$ 0. $\rho_{V}(r)=\operatorname{tr}\left(\mathcal{D}^{\perp}(r)\right)$ is the ensemble density and $\left\|\mathcal{D}^{\perp}(r)\right\|$ is the determinant of $\mathcal{D}^{\perp}(r)$ (eq (33) of Supplementary Note 6).

After a few algebraic operations (For details, see Supplementary Note 6), we arrive at the expression of the correlation energy density (Eq.23),

$$
\mathcal{G}(\mathcal{D}(r))=v_{\mathrm{MS}}^{c}\left[\mathcal{D}-\mathcal{S} \rho_{V}\right]+\mathcal{S} g_{V}
$$

where $g_{V}(r)=\frac{1}{2}\left[g\left(\lambda_{1}(r)\right)+g\left(\lambda_{2}(r)\right)\right]$ is the ensemble correlation energy density and we have defined

$$
v_{\mathrm{MS}}^{c}(r)=\frac{g\left(\lambda_{1}(r)\right)-g\left(\lambda_{2}(r)\right)}{\lambda_{1}(r)-\lambda_{2}(r)}
$$

(Note when $\lambda_{2} \rightarrow \lambda_{1}, v_{\mathrm{MS}}^{c}(r) \rightarrow g^{\prime}\left(\lambda_{1}(r)\right)$.).

Consequently, the Hamiltonian matrix functional under the local density-matrix approximation has the following form, 


$$
\mathcal{H}^{\mathrm{LDMA}}[\mathcal{D}]=\mathcal{T}_{s}+\mathcal{E}_{\mathrm{Hx}}+\int \mathrm{d} r v(r) \mathcal{D}(r)+\mathcal{S} E_{c}[\mathcal{D}]+\int \mathrm{d} r v_{\mathrm{MS}}^{c}(r)\left[\mathcal{D}(r)-\mathcal{S} \rho_{V}(r)\right]
$$

where $E_{c}[\mathcal{D}]=\int \mathrm{d} r g_{V}(r)$ is the ensemble correlation energy. In the two-state system, the correlation energy functional $\mathcal{E}_{c}[\mathcal{D}]$ is divided into two parts: The mean correlation matrix functional, $\mathcal{S} E_{c}[\mathcal{D}]$, directly proportional to the overlap matrix $\mathcal{S}$ and the ensemble correlation energy; The second part is the multi-state correlation matrix functional, $\int \mathrm{d} r v_{\mathrm{MS}}^{c}\left[\mathcal{D}-\mathcal{S} \rho_{V}\right]$, proportional to the difference between $\mathcal{D}$ and the reference density matrix $\mathcal{S} \rho_{V}$. In this sense, $v_{\mathrm{MS}}^{c}(r)$ is named as the multi-state correlation potential, which is invariant under the linear transformation of $\mathcal{D}(r)$ (Eq.9) and is a universal functional that applies to all elements of $\mathcal{D}(r)$. The division is physically meaningful because only the first part contributes to the multi-state energy functional (Eq.20),

$$
E_{\mathrm{MS}}^{\mathrm{LDMA}}[\mathcal{D}]=\operatorname{tr}\left[\mathcal{S}^{-1}\left(\mathcal{T}_{s}+\mathcal{E}_{\mathrm{Hx}}+\int \mathrm{d} r v \mathcal{D}\right)\right]+E_{c}[\mathcal{D}]
$$

while the latter is responsible for the distribution of correlation energy among matrix elements of $\mathcal{H}^{\mathrm{LDMA}}[\mathcal{D}]$ (Supplementary Note 7 ).

Conclusions In this work, we establish the variational principle of MSDFT (Theorem 2) by introducing a Hamiltonian matrix functional, $\mathcal{H}[\mathcal{D}]($ Theorem 1$)$. With a multi-state auxiliary system, an explicit formulation of $\mathcal{H}[\mathcal{D}]$ is presented with the correlation matrix functional, $\mathcal{E}_{c}[\mathcal{D}]$ (Eq.17). An expression of $\mathcal{E}_{c}[\mathcal{D}]$ is given under the local density-matrix approximation (LDMA). In the twostate system, we find that, with a universal correlation potential $v_{\mathrm{MS}}^{c}(r)$, each element of the matrix functional in Eq.30 has an analogous formulation as the KS energy functional, $E_{\mathrm{KS}}\left[\rho_{0}\right]$, suggesting that various functional approximations for the ground state can be applicable to the multistate formulation[4, 23, 42, 43]. Whether the universal potential $v_{\mathrm{MS}}^{c}(r)$ exists for systems of $N_{s}>2$ is subject to further analysis. The multi-state DFT extends the Kohn-Sham energy functional for the ground state to a Hamiltonian matrix functional for a finite number of ground and excited states, paving the way for many future applications in chemistry, biology and materials.

\section{ACKNOWLEDGMENTS}

This work has been partially supported by Shenzhen Municipal Science and Technology Innovation Commission (KQTD2017-0330155106581) and the National Natural Science Foundation of China (21533003). We thank Prof. Wenjian Liu for discussion and insightful comments.
[1] P. Hohenberg and W. Kohn, Inhomogeneous electron gas, Physical Review 136, B864 (1964).

[2] W. Kohn and L. Sham, Self-Consistent Equations Including Exchange and Correlation Effects, Physical Review 140, A1133 (1965).

[3] R. G. Parr and W. Yang, Density Functional Theory of Atoms and Molecules (Oxford University Press, New York, 1989) p. 352.

[4] A. D. Becke, Perspective: Fifty years of densityfunctional theory in chemical physics, Journal of Chemical Physics 140, 1 (2014).

[5] E. Runge and E. K. Gross, Density-Functional Theory for Time-Dependent Systems, Physical Review Letters 52, 997 (1984).

[6] R. E. Stratmann, G. E. Scuseria, and M. J. Frisch, An efficient implementation of time-dependent densityfunctional theory for the calculation of excitation energies of large molecules, Journal of Chemical Physics 109, 8218 (1998).

[7] M. A. Marques, C. A. Ullrich, F. Nogueira, A. Rubio, K. Burke, and E. K. Gross, Time-dependent density functional theory, The Lecture Notes in Physics (Springer, 2006) p. 603.
[8] R. O. Jones, Density functional theory: Its origins, rise to prominence, and future, Reviews of Modern Physics 87, 897 (2015).

[9] B. Kaduk, T. Kowalczyk, and T. Van Voorhis, Constrained density functional theory, Chemical Reviews 112, 321 (2012).

[10] F. Caruso, D. R. Rohr, M. Hellgren, X. Ren, P. Rinke, A. Rubio, and M. Scheffler, Bond breaking and bond formation: How electron correlation is captured in manybody perturbation theory and density-functional theory, Physical Review Letters 110, 1 (2013).

[11] J. Hermann, R. A. DiStasio, and A. Tkatchenko, FirstPrinciples Models for van der Waals Interactions in Molecules and Materials: Concepts, Theory, and Applications, Chemical Reviews 117, 4714 (2017).

[12] Z. H. Yang, A. Pribram-Jones, K. Burke, and C. A. Ullrich, Direct Extraction of Excitation Energies from Ensemble Density-Functional Theory, Physical Review Letters 119, 1 (2017).

[13] A. K. Theophilou, The energy density functional formalism for excited states, Journal of Physics C: Solid State Physics 12, 5419 (1979).

[14] N. D. Mermin, Thermal Properties of the Inhomogeneous Electron Gas, Physical Review 137, 1441 (1965). 
[15] E. K. Gross, L. N. Oliveira, and W. Kohn, Rayleigh-Ritz variational principle for ensembles of fractionally occupied states, Physical Review A 37, 2805 (1988).

[16] E. K. Gross, L. N. Oliveira, and W. Kohn, Densityfunctional theory for ensembles of fractionally occupied states. I. Basic formalism, Physical Review A 37, 2809 (1988).

[17] N. I. Gidopoulos, P. G. Papaconstantinou, and E. K. Gross, Spurious Interactions, and Their Correction, in the Ensemble-Kohn-Sham Scheme for Excited States, Physical Review Letters 88, 4 (2002).

[18] T. Gould and S. Pittalis, Hartree and Exchange in Ensemble Density Functional Theory: Avoiding the Nonuniqueness Disaster, Physical Review Letters 119, 1 (2017).

[19] T. Gould and S. Pittalis, Density-Driven Correlations in Many-Electron Ensembles: Theory and Application for Excited States, Physical Review Letters 123, 16401 (2019).

[20] E. Fromager, Individual Correlations in Ensemble Density Functional Theory: State- And Density-Driven Decompositions without Additional Kohn-Sham Systems, Physical Review Letters 124, 243001 (2020).

[21] T. Gould, G. Stefanucci, and S. Pittalis, Ensemble Density Functional Theory: Insight from the FluctuationDissipation Theorem, Physical Review Letters 125, 233001 (2020).

[22] T. Gould and L. Kronik, Ensemble generalized KohnSham theory: The good, the bad, and the ugly, Journal of Chemical Physics 154, 1 (2021).

[23] N. Mardirossian and M. Head-Gordon, Thirty years of density functional theory in computational chemistry: An overview and extensive assessment of 200 density functionals, Molecular Physics 115, 2315 (2017).

[24] J. Gao, A. Grofe, H. Ren, and P. Bao, Beyond KohnSham Approximation: Hybrid Multistate Wave Function and Density Functional Theory, Journal of Physical Chemistry Letters 7, 5143 (2016).

[25] A. Cembran, L. Song, Y. Mo, and J. Gao, Diabatic Coupling, and Their Use in Valence Bond, Journal of Chemical Theory and Computation 5, 2702 (2009).

[26] H. Ren, M. R. Provorse, P. Bao, Z. Qu, and J. Gao, Multistate Density Functional Theory for Effective Diabatic Electronic Coupling, Journal of Physical Chemistry Letters 7, 2286 (2016).

[27] A. Grofe, Z. Qu, D. G. Truhlar, H. Li, and J. Gao, Diabatic-At-Construction Method for Diabatic and Adiabatic Ground and Excited States Based on Multistate Density Functional Theory, Journal of Chemical Theory and Computation 13, 1176 (2017).

[28] R. Zhao, C. P. Hettich, and X. Chen, Minimal-activespace multistate density functional theory for excitation energy involving local and charge transfer states, npj Computational Materials 7, 1 (2021).

[29] R. Zhao, A. Grofe, Z. Wang, P. Bao, X. Chen, W. Liu, and J. Gao, Dynamic-then-Static Approach for Core Ex- citations of Open-Shell Molecules, The Journal of Physical Chemistry Letters 12, 7409 (2021).

[30] W. L. Chan, T. C. Berkelbach, M. R. Provorse, N. R. Monahan, J. R. Tritsch, M. S. Hybertsen, D. R. Reichman, J. Gao, and X. Y. Zhu, The quantum coherent mechanism for singlet fission: Experiment and theory, Accounts of Chemical Research 46, 1321 (2013).

[31] A. Cembran, M. R. Provorse, C. Wang, W. Wu, and J. Gao, The third dimension of a more O'Ferrall-Jencks diagram for hydrogen atom transfer in the isoelectronic hydrogen exchange reactions of $(\mathrm{PhX}) 2 \mathrm{H} \bullet$ with $\mathrm{X}=\mathrm{O}$, $\mathrm{NH}$, and $\mathrm{CH} 2$, Journal of Chemical Theory and Computation 8, 4347 (2012).

[32] A. D. Becke, A. Savin, and H. Stoll, Extension of the local-spin-density exchange-correlation approximation to multiplet states, Theoretica Chimica Acta 91, 147 (1995).

[33] W. Liu and M. R. Hoffmann, Sds: the 'static-dynamicstatic' framework for strongly correlated electrons, in Isaiah Shavitt: A Memorial Festschrift from Theoretical Chemistry Accounts, edited by R. Shepard, R. M. Pitzer, and T. Dunning (Springer Berlin Heidelberg, Berlin, Heidelberg, 2016) pp. 141-152.

[34] M. Levy, Universal variational functionals of electron densities, first-order density matrices, and natural spinorbitals and solution of the v-representability problem, Proceedings of the National Academy of Sciences of the United States of America 76, 6062 (1979).

[35] E. H. Lieb, Density functionals for coulomb systems, International Journal of Quantum Chemistry 24, 243 (1983).

[36] T. Gould, L. Kronik, and S. Pittalis, Charge transfer excitations from exact and approximate ensemble KohnSham theory, Journal of Chemical Physics 148, 1 (2018).

[37] B. C. Hall, Lie groups, Lie algebras and representations: An elementary introduction, Graduate Texts in Mathematics (Springer, 2015) p. 453.

[38] L. D. Landau and E. M. Lifshitz, Quantum mechanics: Non-relativistic theory, Course of Theoretical Physics, Vol. 3 (Pergamon Press, 1977) p. 679.

[39] R. McWeeny, Some recent advances in density matrix theory, Reviews of Modern Physics 32, 335 (1960).

[40] K. J. Oosterbaan, A. F. White, and M. Head-Gordon, Non-orthogonal configuration interaction with single substitutions for the calculation of core-excited states, Journal of Chemical Physics 149 (2018).

[41] I. Gohberg, S. Goldberg, and M. A. Kaashoek, Classes of Linear Operators: Volumn 1 (Birkhauser, 1991) p. 468.

[42] C. Lee, W. Yang, and R. G. Parr, Development of the Colic-Salvetti correlation-energy formula into a functional of the electron density, Physical Review B 37, 785 (1988).

[43] J. P. Perdew, K. Burke, and M. Ernzerhof, Generalized gradient approximation made simple, Physical Review Letters 77, 3865 (1996). 